\title{
Arbeitsfähig bleiben - Balance halten - Einsichten gewinnen
}

\author{
Wie Coaching und Supervision Qualität für Organisationen \\ sichern
}

\section{Erik de Haan}

Online publiziert: 21. Oktober 2016

(C) Der/die Autor(en) 2016. Dieser Artikel ist eine Open-Access-Publikation.

Zusammenfassung Der Autor befasst sich damit, wie Management, Coaching und Supervision zusammenarbeiten können, um die Risiken einer starken Führungsorientierung zu mildern. Er untersucht, wie eine Führungskraft, die ihr eigenes Führungsverhalten ändern und ihre verletzliche ,Schattenseite“ integrieren will, ihre Qualität als Führungskraft erhalten und verbessern kann. Wie können Führungskräfte in der Arbeit mit einem Coach, der auch ihre Bedürftigkeit, Verletzlichkeit, Zweifel und Selbsttäuschung anspricht, ein Führungshandeln entwickeln, das die Qualität ihrer Führung gewährleistet? Und wie können Coaches ihre Servicequalität durch Supervision mit einem Außenstehenden gewährleisten? Es zeigt sich, dass Supervision oft einmalige Gelegenheiten bietet, verborgene Dynamiken der Führungspraxis zu erfassen und zu bedenken.

Schlüsselwörter Führung · Führungscoaching · Supervision · Qualitätssicherung

\section{Keeping fit, maintaining balance, gaining insight}

How coaching and supervision provide quality assurance for organisations

\begin{abstract}
In today's fast paced, interconnected, and mercilessly competitive business world, senior executives have to push themselves and others hard. In order to succeed, leaders have to live the paradox of closely attending to and following others as deeply as they lead. They have to listen well to others, understand their concerns, give them personal support, and at the same time motivate them for results or take decisions on their behalf. This article explores how executive coaching together with the provision of supervision for coaches can help restore and maintain balance and so provide quality assurance for organisations. Some of the work that these coaches
\end{abstract}

Prof. Dr. E. de Haan $(\bowtie)$

Ashridge House, Berkhamsted, HP41NS, Hertfordshire, UK

E-Mail: Erik.deHaan@ashridge.hult.edu 
and supervisors do is simply noticing the shadow sides that leaders have forgotten about or for many reasons prefer not to consider. It is the coach's (and supervisor's) task to bring back awareness of vulnerability or neediness, corruptibility or hubris, depending on the highly personal contents of the leader's shadow.

Keywords Leadership · Executive Coaching · Supervision · Quality Monitoring

\section{Die Schattenseite von Führung: die Verletzlichkeit in der Führungsfunktion}

In der heutigen schnelllebigen, vernetzten und gnadenlos wettbewerbsorientierten Geschäftswelt müssen Führungskräfte sich selbst und andere hart antreiben. Um erfolgreich zu sein, müssen Führungskräfte das Paradox handhaben, die Anderen sowohl engmaschig zu begleiten als auch sie zu führen. Sie müssen gut zuhören, die Anliegen anderer verstehen, ihnen persönliche Unterstützung geben, sie aber gleichzeitig motivieren, Ergebnisse zu erzielen, und überdies Entscheidungen in ihrem Namen treffen.

Gerade Führungskräfte, die sich gut an diese Bedingungen anpassen und die einen harten und unnachgiebigen Fokus auf Wettbewerbsvorteile entwickeln können, sind am stärksten gefährdet, wenig hilfreiche und letztlich unproduktive Muster des Forderns, der Sturheit oder einer übermäßigen Aktivität zu entwickeln. Statt offen für Möglichkeiten und Mehrdeutigkeit zu sein und in kreative Auseinandersetzungen mit sich selbst und anderen einzutreten, werden diese Führungskräfte hartnäckig, gereizt, artikulieren sich nicht oder überintensiv. Sie werden zu einer Karikatur ihrer selbst und ,übersteuern“. Meistens sind dies Qualitäten, auf die sie besonders vertraut haben, um an die Spitze zu kommen und hervorragende Ergebnisse zu erzielen, - Qualitäten, die unter Stress und Druck zu überschießenden, wenig hilfreichen „Hamsterrädern“ werden und die zu geschäftlichen wie persönlichen Katastrophen (Claxton et al. 2015) führen. Bisher hoch leistungsfähige Führungskräfte sehen sich plötzlich mit Beziehungsabbrüchen, strategischen Fehlern oder den Risiken einer Entgleisung konfrontiert.

Sich mit einem Führungsanspruch zu zeigen, erzeugt immer einen Riss in sich selbst: eine Kluft zwischen einer sonnigen, aktiven, konstruktiven bzw. aggressiven Seite in sich selbst, voll des Ehrgeizes, etwas zu schaffen und sich zu beweisen; und einer zweifelnden, pessimistischen, bedürftigen, verletzlichen, vorsichtigen und besorgten Seite, die sich nach Verbindung mit sich selbst und anderen sehnt. Diese Schattenseite ist daher stets ein wesentlicher Bestandteil von Führung. Dieser Riss und das Wechselspiel von Hell und Dunkel, die diesen Prozess begleiten, können sehr subtil sein; man kann z. B. eine sehr fürsorgliche Seite von sich selbst in die Führungsrolle einbringen, eine besondere Wärme, Fürsorge, Achtsamkeit und Aufmerksamkeit. Auch in solchen Fällen existiert unweigerlich eine ganze ,andere“ Seite unserer Persönlichkeit, die wir zurückdrängen, um eine solche Führungsrolle durchzutragen.

Im Falle einer sehr fürsorglichen, besorgten, warmen Führungskraft kann es also eine Seite von sich selbst geben, die mit Konflikten, Ärger, Selbstwert zu tun hat, die 
es gilt, unten zu halten. Das „Führungsschatten-Phänomen“ ist in allen Führungspositionen ständig präsent. Um offensiv anzubieten oder auf „Vollgas“ zu schalten, müssen andere Aspekte zurückgelassen werden, zurückgedrängt und verworfen, irgendwo in der Dunkelheit unseres Erlebens, einschließlich des Selbsterlebens.

\section{Fallbeispiel}

Wir haben uns an unsere tägliche Dosis von Unternehmensskandalen an der Spitze von Organisationen gewöhnt, deren Produkte und Dienstleistungen wir nur allzu gerne kaufen. Ein Beispiel vom 21. Juli 2015: der CEO von Toshiba ist zusammen mit seinem Vorgänger und einem Großteil der Führungskräfte zurückgetreten. Die Financial Times schreibt: „Ein Gremium externer Anwälte und Wirtschaftsprüfer sagte am Montag, es habe ein ,systematischer“ und ,gezielter“ Versuch stattgefunden, Gewinnzahlen aufzublasen, und dies in einer Unternehmenskultur, in der Mitarbeitende Angst hatten, Vorgesetzten zu widersprechen, die unrealistische Ergebnisziele einforderten. “ Der Geschäftsführer Herr Tanaka sagte in einer Pressekonferenz nach einer zerknirschten 15-Sekunden-Verbeugung, dass er ,eine Generalüberholung unseres Managementteams für notwendig halte, um unser Unternehmen neu aufzubauen." Tanaka waren die bewusst übertrieben ausgewiesenen Gewinne ganz offensichtlich bekannt, und er hatte keine Maßnahmen ergriffen, um die falsche Buchhaltung zu beenden. Das Top-Management sollte „Herausforderungen“ oder Ertrags-Verbesserungs-Ziele in monatlichen Treffen mit den Leitern der In-house-Firmen sowie der Tochtergesellschaften festsetzen, und dieses Streben nach Verbesserung hatte letztlich das Gegenteil dessen hervorgebracht, was erreicht werden sollte. Tanaka trat mit seinem Vorgänger und fünf weiteren Vorstandsmitgliedern zurück. Diejenigen, die Andere mit Forderungen nach blinder Loyalität und nach Erreichung unmöglicher Ziele unter Druck gesetzt hatten, verlassen jetzt die Firma mit mehr als einer Milliarde Dollar ungeklärter Kosten und noch größeren Schäden an der Marke.

Die große Herausforderung für Führungskräfte kann durch das gleiche Paradox von Führung zusammengefasst werden, mit dem dieser Artikel begann. Es ist die Kunst, proaktiv auf eine „Führungsagenda“ zu fokussieren und dies in Balance zu bringen mit dem „Schutt“, den diese Agenda mit sich bringt: den Widersprüchen, Zweifeln und Schwachstellen - mit dem dunklen Schatten von Führung sowohl in der Persönlichkeit der Führungskraft als auch im Unternehmen.

\section{Qualitätssicherung für Führungskräfte - wie Coaches und Organisationentwickler helfen können}

Die Schattenseite spielt eine Rolle in jeder Form der Führung; doch solange der Job nur einen Teil der Zeit in Anspruch nimmt und Führungskräfte einen willensstarken, konfrontativen Ehepartner sowie durchsetzungsfähige Kollegen haben, die sie an ihre menschliche Zerbrechlichkeit und Fehlbarkeit erinnern, kann der Führungsschatten so verarbeitet werden, dass er nicht allzu viele Probleme verursacht. Leider sind viele unserer Industriekapitäne und politischen Führer nicht in einer solch glücklichen Lage. Sie sind engagiert, ja hingebungsvoll an ihren Job, widmen ihm exorbitant viel Zeit und Mühe, und sie werden selten von den Menschen in ihrer unmittelbaren 
Umgebung kritisiert oder in Frage gestellt. Wie sollen Führungskräfte unter solchen Bedingungen unverbraucht und ausgeglichen bleiben, bzw. offen und selbstkritisch ihre eigenen fest verwurzelten Überzeugungen reflektieren?

Ich glaube, dass anspruchsvolle, freimütige und offene Beratung von (Top-) Führungskräften ihnen helfen kann, sie an ihren sehr persönlichen Führungsschatten zu erinnern und an die Tatsache, dass auch bei ihnen (verdeckte) Zweifel, Bedürfnisse und Schwachstellen Bestandteil ihres Führungsverhaltens sind. Sie mögen dies nicht so in der Öffentlichkeit sagen, aber auf einer bestimmten Ebene wissen sie selbst, wie schwach ihre Führung in einem größeren Kontext erscheinen kann (De Haan und Kasozi 2014). Dieses offene und anspruchsvolle In-Frage-Stellen von Managern und Führungskräften wird durch Organisationsentwickler und Führungscoaches ermöglicht, wenn sie die beträchtlichen Honorare, die ihnen bezahlt werden, wert sind (Nelson und Hogan 2009).

Ein Teil der Arbeit, die Coaches und Berater tun, besteht darin, die Schattenseiten zu erkennen oder zu erraten, welche die Führungskräfte vergessen haben oder aus vielen Gründen lieber nicht berücksichtigen. Es ist die Aufgabe des Coachs, Verletzlichkeit oder Bedürftigkeit, Bestechlichkeit oder Hybris, entsprechend den sehr persönlichen Inhalten des Führungsschattens, wieder ins Bewusstsein zu bringen. Coaching wirkt ausgleichend und kümmert sich um die „Fitness des Machens“ genau dadurch, dass sie Einsicht erzeugen in die empfindlichsten und gefährdeten Anteile der Führungskraft.

In einer anderen Form der Selbstführung ist wiederum vom Coach oder Berater gefordert, ehrlich und furchtlos ihre persönliche Wahrheit Mächtigen gegenüber (De Haan 2006) auszusprechen und frei und unabhängig mit den Führungskräften deren aktuellen Führungsfragen zu reflektieren. Die Frage stellt sich: Wie bleiben Coaches für die Praxis fit und stellen sicher, dass sie ihren Kunden mit dem erforderlichen $\mathrm{Maß}$ an Klarheit und Stabilität gegenübertreten? Die Antwort auf diese Frage lautet: Supervision.

\section{Qualitätssicherung für Coaches - wie Supervisor/innen jene unterstützen, die Führungskräfte unterstützen}

Zugang zu einem Führungscoaching gilt längst nicht mehr als Privileg, das auf die Elite einer Organisation beschränkt ist: In den letzten zehn Jahren ist Coaching zu einer Entwicklungsmaßnahme bei akuten Anforderungen geworden, eingesetzt in einem breiten Spektrum betrieblicher und technischer Settings. Dieser erweiterte Zugang stellt die Personalentwicklung vor die Herausforderung, sicherzustellen, dass Coaching für den jeweiligen Zweck geeignet ist. Dies ist keine einfache Aufgabe, da die überwiegende Mehrheit der Coaching-Beziehungen durch strikte Vertraulichkeit gekennzeichnet ist. Noch vor wenigen Jahren war es leicht, mit Empfehlungen und Erfahrungen als Coach einzusteigen. Qualifikationen waren kein Thema, und nur sehr wenige Organisationen fragten nach Ausbildung oder gar kontinuierlicher beruflicher Weiterbildung.

All das ändert sich: Die meisten Großunternehmen setzen mittlerweile interne wie externe Coaches ein, von denen erwartet wird, dass sie von einer anerkann- 
ten Institution ausgebildet und akkreditiert sind. In vielerlei Hinsicht spiegelt diese zunehmende Anforderung an die Professionalität von Coaches die Erwartung, dass auch Führungskräfte in der Wirtschaft sich professionell (weiter) zu entwickeln haben.

Diese wachsende Professionalisierung von Coaching und Unternehmensführung hat zum Wachstum von Business-Schulen beigetragen, mit MSCs in Organisationsberatung oder Coaching, neben den traditionellen Master- und Doktorats-Programmen. Allerdings kann eine formale Coaching-Qualifikation nicht per se als Beweis für Professionalität und Kompetenz genommen werden. Coaching ist eine höchst anspruchsvolle und einsame Tätigkeit, voller Kämpfe darum, eine wahrhaftige und zugleich sensible Sprache zu finden, die geeignet ist, Macht, Zweifel, ethische Dilemmata oder die Verführung, ein dysfunktionales Führungsverhalten zu stützen, anzusprechen (De Haan 2008; De Haan und Carroll 2014a, 2014b).

Aus diesem Grund erwarten wir von Coaches und Organisationsberater/innen, regelmäßig Supervision in Anspruch zu nehmen. Z. B. verlangen wir von unseren eigenen Ashridge Coaches nach Abschluss der Ausbildung Nachweise kontinuierlicher Supervision, wenn sie ihre berufliche Akkreditierung erhalten wollen. Supervision ist kein „,nützlicher Zusatz“, sondern eine wesentliche Voraussetzung für die Aufrechterhaltung der Qualität, Kompetenz und Professionalität eines Coachs (De Haan und Birch 2010) und Organisationsberaters (De Haan und Birch 2011). Supervision von Coaching findet entweder in Gruppen oder auf einer 1:1 Basis statt. Sie soll den Coach bestmöglich unterstützen, damit er seinen Kunden dabei helfen kann, ihre Führungsschatten zu erkennen sowie die Verantwortung für ihre Entscheidungen zu übernehmen. Supervision als entwicklungsorientierter Beratungsprozess verfolgt das Ziel, die Qualität der Coaches in der Arbeit mit ihren Kunden zu begleiten und zu verbessern. Je besser der Kontakt zu den eigenen emotionalen und intellektuellen Ressourcen gehalten werden kann, desto besser werden Coaches auch ihren Kunden helfen können.

Was bedeutet das in der Praxis? Das wesentliche Merkmal von Supervision ist das regelmäßige Zur-Verfügung-Stellen eines vertraulichen Raumes, in dem Coaches ihre berufliche Praxis reflektieren können. Coaches sind oft sehr damit beschäftigt, ihr Coaching mit anderen Berufsrollen zu kombinieren, die wiederum mit Anforderungen und Aufgaben außerhalb der Arbeit balanciert werden müssen. Auch wenn es immer eine Herausforderung darstellt, sich eine Auszeit von einem hektischen Zeitplan zu nehmen, sind die Vorteile für den Coach und ihre Kunden potenziell enorm.

\section{Fallbeispiel}

Ein erfahrener Coach arbeitete mit einem Kunden, der gerade Vater wurde und unter starkem Druck stand, sowohl zu Hause als auch in seiner Führungsrolle. Der Coach arbeitete schon seit längerem mit dem Kunden, und sie hatten eine stabile Vertrauensbasis aufgebaut. In den ersten Sitzungen hatte der Kunde kaum Emotionen geäußert, aber jetzt zeigte er immense Angst, tiefe Wut und ein Gefühl der Hilflosigkeit. Der Coach fühlte sich von den starken Gefühlen seines Kunden überwältigt und traute seiner eigenen Kompetenz nicht, mit diesem Ausmaß an Emotionen zu arbeiten. Zugleich erkannte er, dass für den Kunden ihre vertraute Beziehung einer der 
wenigen Orte war, wo er seiner Verzweiflung Ausdruck verleihen konnte. Während der Supervision untersuchte der Coach seine eigene emotionale Reaktion auf seinen Klienten und entdeckte zu seiner Überraschung einen stark beschützenden Impuls ihm gegenüber. Mit dieser Erkenntnis und der Ermutigung seines Supervisors fühlte er sich stark genug, um seinem Klienten eine klare Grenze zu setzen, die es diesem ermöglichte, seine Gefühle in einer distanzierteren Weise zu erkunden. Supervisor und Coach einigten sich, dass er, wenn er sich oder seinen Kunden als überwältigt erlebte, den Supervisor wieder kontaktieren würde, notfalls zwischen den Sitzungen.

Interessanterweise ist die Art der Qualitätssicherung, die Supervision für Coaches bietet, vergleichbar mit der Qualitätssicherungs-Funktion der Coaches für Führungskräfte. Genau wie Coaches ihren Kunden helfen Supervisor/innen Coaches, ehrlicher und tiefgehender zu reflektieren sowie die „Schattenaspekte“ ihrer Dienstleistung zu erkennen, zu akzeptieren und handhabbar zu machen - wie etwa bestimmte Führungskräfte nicht zu konfrontieren, große Geschenke von Kunden zu akzeptieren oder intuitiv und wenig hilfreich auf den Führungsschatten zu reagieren (um nur einige zu nennen).

Supervision ist oft eine letzte Gelegenheit, tief versteckter, nicht hilfreicher Dynamiken in Organisationen gewahr zu werden. Ich habe oft bemerkt, dass ein etwas gestelzter, ungeschickter oder aufschlussreicher Moment in der Supervision auf ein Muster verwies, das letztlich den Kern des problematischen Verhaltens einer Führungskraft berührte. Und auch wenn zunächst nur in der Supervision bemerkt, half es dem Coach, zurückzugehen, zu reagieren und einen positiven Unterschied für viele Beteiligte $\mathrm{zu}$ machen.

\section{Einige ethische Überlegungen zur Qualitätssicherung für Führungskräfte und Coaches}

Ähnlich wie ein Führungsfokus in schädliche Übersteuerung und Entgleisung gehen kann, gibt es auch erhebliche Risiken in Coaching und Supervision - ganz abgesehen von der Gefahr der einfach nicht richtig erfassten Praxis der eigenen Klienten -, in Übersteuerung zu gehen.

Berglas (2002) fasst die „sehr realen Gefahren des Führungscoachings“ zusammen, die seiner Ansicht nach oft durch einen persönlichen Mangel des Coachs an psychologischem Verständnis verschärft werden. Er gibt Fallbeispiele von Coaches, die

- die Situation falsch einschätzen,

- den Status quo verschlimmern und

- ihre eigene Macht missbrauchen.

Weitere ethische Herausforderungen im Coaching und in anderen Beratungsberufen erwachsen aus folgenden Situationen:

1. die vielen neuen Coaches, oft (interne) Berater, HR-Direktoren und CEOs, die immer noch diesen Beruf mit nur minimaler Ausbildung beginnen, 
2. sehr geringe Verhandlungsmacht der Kunden, die eine Vielzahl von sehr kleinen Kontrakten verhandeln,

3. ein Mangel an Regulierung in Beratungsberufen und Coaching,

4. Gefühle der Scham, Angst und der daraus resultierende Kundenschutz, wenn es Schwierigkeiten oder Bedenken gibt,

5. eine gewisse „Taubheit“ oder Widerstand gegen die externe Qualitätssicherung, insbesondere mit erfahrenen Coaches und Supervisor/innen.

Meiner Meinung nach sind die folgenden Praktiken eine relativ „zahnlose“ Qualitätssicherung:

- Verwaltungssysteme, die nur Kästchen ankreuzen oder in die „Fakten“ der Praxis schauen, nicht in deren gelebte Erfahrung.

- Gedankenlose Anwendung von Verhaltenskodizes als „Dilemma-Ethik“ und über bloße ethische Vignetten, die wenig zu angewandter Ethik im wirklichen Leben beitragen.

- „Moralische Argumentation“, die auf „moralisches Handeln“ nachweislich wenig Einfluss hat. Es ist lange bekannt, dass im Wesentlichen zwei ethische Menschen in allen von uns stecken: (1) das zukünftige ethische Ich, das eines Tages diese Dinge tun wird, die ich für andere empfehle, und (2) das heutige ethische Ich, das aus einem anderen Set von Standards heraus reagiert (Carroll und Shaw 2012).

- „Selbst-lose“ oder „,beziehungslose“ Ethik lässt die Prämisse vergessen, die jeder Ethik und allen moralischen Codes zugrunde liegt, die sogenannte ,goldene Regel“, zuerst formuliert von Confucius, „Behandle andere so, wie du von ihnen behandelt werden willst"“.

Schließlich noch einige Aspekte der Qualitätssicherung durch Coaching und Supervision, die, so glaube ich, positive Auswirkungen in der Praxis haben:

1. Externe Qualitätssicherung scheint zuverlässiger als Peer-Qualitätssicherung; externes Coaching und externe Supervision sind für die Qualitätssicherung internem Coaching und interner Supervision vorzuziehen.

2. Die Marktkräfte, die Dreiecksbeziehung und die allgemein üblichen befristeten Kontrakte spielen eine stabilisierende Rolle und bieten Schutz vor Überschreitungen.

3. Supervision wird zurzeit noch am häufigsten in der Ausbildung eingesetzt, könnte jedoch eine wichtigere Rolle für den Einstieg (fertig ausgebildeter) Coaches und auch für sehr erfahrene Coaches spielen können, die beiden am stärksten gefährdeten Gruppen.

\section{Fallbeispiel}

Ein Team von Coaches, die mit „High Potential“ Führungskräften einer Regierungsabteilung gearbeitet hatten, traf sich zur Gruppensupervision. Im Laufe der Zeit wurde deutlich, dass es in vielen der Coaching-Sitzungen um die schlechten Beziehungen der Coachees zum Führungsteam ging, wofür viele Coachees dem Senior Management die Schuld zuwiesen, das zurückhaltend und uninteressiert sei. Einige der Coaches sahen die Geschäftsleitung ähnlich und wollten, dass der Supervisor die 
Bedenken an das Management über die Personalentwicklung zurückmelden möge. Der Supervisor half den Coaches zu erkennen, dass sie die Sicht ihrer Klienten übernommen hatten und Teil eines „Parallelprozesses“ (De Haan 2012) waren. Er half dann den Coaches, sowohl ihre eigene Rolle zu überdenken als auch die persönliche Verantwortung der Coachees für ihre Interaktion mit dem Senior Management.

Es ist ermutigend, dass immer mehr Führungskräfte über ihr Vertrauen zu ihren Coaches sprechen und dass in ähnlicher Weise Coaches die Unterstützung durch Supervision kommunizieren sowie Entwicklungsbeauftragte etwa bei größeren Projekten im Voraus vorsehen. Die meisten Ashridge-Kunden stimmen mit diesem Ansatz und unserer Ansicht überein, dass (Gruppen-)Supervision integraler Bestandteil des Qualitätssicherungsprozesses ist - ähnlich wie andere Elemente, etwa vereinbarte Sitzungen und Evaluation.

Um zusammenzufassend Gary Embelton (2002) zu paraphrasieren: Ethik bedeutet nicht, Probleme zu lösen. Es geht um Fragen gelebter Beziehungen. Halten Sie Kontakt zu Ihrem eigenen Schmerz und - was auch immer geschieht - stülpen Sie diesen Schmerz nicht anderen über. Dies ist die eigentliche Aufgabe der Supervision: die dünnwandige Respektabilität des Coachings zu durchbrechen und die oft brutalen, destruktiven und verrückten Kräfte zwischen Führungskraft, Coach und Supervisor/in zu erkunden.

Danksagung Ich möchte einen ganz herzlichen Dank sagen an Frau Dr. Michaela Judy von der Wiener Volkshochschule für ihre sorgfältige Übersetzung dieses Artikels.

Open Access Dieser Artikel wird unter der Creative Commons Namensnennung 4.0 International Lizenz (http://creativecommons.org/licenses/by/4.0/deed.de) veröffentlicht, welche die Nutzung, Vervielfältigung, Bearbeitung, Verbreitung und Wiedergabe in jeglichem Medium und Format erlaubt, sofern Sie den/die ursprünglichen Autor(en) und die Quelle ordnungsgemäß nennen, einen Link zur Creative Commons Lizenz beifügen und angeben, ob Änderungen vorgenommen wurden.

Open access funding provided by VU University Amsterdam.

\section{Literatur}

Berglas, S. (2002). The very real dangers of executive coaching. Harvard Business Review, 80, 86-92.

Carroll, M., \& Shaw, E. (2012). Ethical maturity in the helping professions. Victoria: PsychOz Publications.

Claxton, G., Owen, D., \& Sadler-Smith, E. (2015). Hubris in leadership: a peril of unbridled intuition? Leadership, 11(1), 57-78.

De Haan, E. (2006). Fearless consulting. Chichester: Wiley.

De Haan, E. (2008). I doubt therefore I coach - critical moments in coaching practice. Consulting Psychology Journal: Practice and Research, 60(1), 91-105.

De Haan, E. (2012). Supervision in action: a relational approach to coaching and organization supervision. Columbus: McGraw-Hill, Open University Press.

De Haan, E., \& Birch, D. (2010). Quality control for coaching. The Training Journal, August, 71-74.

De Haan, E., \& Birch, D. (2011). Supervision for consultants. The Training Journal, January, 63-67.

De Haan, E., \& Carroll, M. (2014a). Moral Lessons - Part 1: what would you do? Coaching @ Work, 9, 37-39.

De Haan, E., \& Carroll, M. (2014b). Moral Lessons - Part 2: further guidance. Coaching @ Work, 9, 46-50.

De Haan, E., \& Kasozi, A. (2014). The leadership shadow: how to recognize and avoid derailment, hubris and overdrive. London: Kogan Page. 
Embelton, G. (2002). Dangerous liaisons and shifting boundaries in psychoanalytic perspectives on supervision. In M. McMahon \& W. Patton (Hrsg.), Supervision in the helping professions: a practical approach (S. 119-130). French's Forest: Pearson.

Nelson, E., \& Hogan, R. (2009). Coaching on the dark side. International Coaching Psychology Review, 4, 7-19.

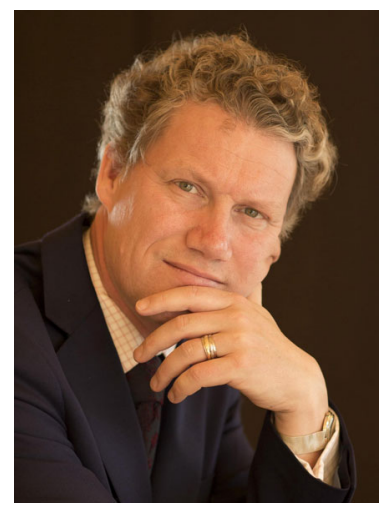

Prof. Dr. Erik de Haan Professor für Organisationsentwicklung, VU University Amsterdam, Direktor desAshridge Centre for Coaching, Ashridge Executive Education at Hult International Business School, Ashridge House, Berkhamsted. Internet: http://www.ashridge.org.uk/ erikdehaan. 